\title{
BMJ Open Lifestyle and vascular risk effects on MRI-based biomarkers of Alzheimer's disease: a cross-sectional study of middle-aged adults from the broader New York City area
}

\author{
Lisa Mosconi, ${ }^{1,2,3}$ Michelle Walters, ${ }^{3}$ Joanna Sterling, ${ }^{4}$ Crystal Quinn, ${ }^{2}$ \\ Pauline McHugh, ${ }^{2}$ Randolph E Andrews, ${ }^{5}$ Dawn C Matthews, ${ }^{5}$ Christine Ganzer, ${ }^{6}$ \\ Ricardo S Osorio, ${ }^{2}$ Richard S Isaacson, ${ }^{1}$ Mony J De Leon, ${ }^{2}$ Antonio Convit ${ }^{2}$
}

To cite: Mosconi L, Walters M, Sterling J, et al. Lifestyle and vascular risk effects on MRI-based biomarkers of Alzheimer's disease: a crosssectional study of middle-aged adults from the broader New York City area. BMJ Open 2018;8:e019362. doi:10.1136/ bmjopen-2017-019362

- Prepublication history and additional material for this paper are available online. To view these files, please visit the journal online (http://dx.doi. org/10.1136/bmjopen-2017019362).

Received 5 September 2017 Revised 29 November 2017 Accepted 8 December 2017

Check for updates

For numbered affiliations see end of article.

Correspondence to

Dr Lisa Mosconi;

lim2035@med.cornell.edu

\section{ABSTRACT}

Objective To investigate the effects of lifestyle and vascular-related risk factors for Alzheimer's disease (AD) on in vivo MRI-based brain atrophy in asymptomatic young to middle-aged adults.

Design Cross-sectional, observational.

Setting Broader New York City area. Two research centres affiliated with the Alzheimer's disease Core Center at New York University School of Medicine.

Participants We studied 116 cognitively normal healthy research participants aged 30-60 years, who completed a three-dimensional T1-weighted volumetric MRI and had lifestyle (diet, physical activity and intellectual enrichment), vascular risk (overweight, hypertension, insulin resistance, elevated cholesterol and homocysteine) and cognition (memory, executive function, language) data. Estimates of cortical thickness for entorhinal (EC), posterior cingulate, orbitofrontal, inferior and middle temporal cortex were obtained by use of automated segmentation tools. We applied confirmatory factor analysis and structural equation modelling to evaluate the associations between lifestyle, vascular risk, brain and cognition. Results Adherence to a Mediterranean-style diet (MeDi) and insulin sensitivity were both positively associated with MRI-based cortical thickness (diet: $\beta_{s} \geq 0.26$, insulin sensitivity $\left.\beta_{\mathrm{s}} \geq 0.58, P \leq 0.008\right)$. After accounting for vascular risk, $E C$ in turn explained variance in memory $(P \leq 0.001)$. None of the other lifestyle and vascular risk variables were associated with brain thickness. In addition, the path associations between intellectual enrichment and better cognition were significant $\left(\beta_{s} \geq 0.25 \mathrm{P} \leq 0.001\right)$, as were those between overweight and lower cognition $\left(\beta_{s} \geq-0.22\right.$, $\mathrm{P} \leq 0.01)$.

Conclusions In cognitively normal middle-aged adults, MeDi and insulin sensitivity explained cortical thickness in key brain regions for $A D$, and EC thickness predicted memory performance in turn. Intellectual activity and overweight were associated with cognitive performance through different pathways. Our findings support further investigation of lifestyle and vascular risk factor modification against brain ageing and AD. More studies

\section{Strengths and limitations of this study}

One of the key strengths of this study is the availability of a well-characterised population of asymptomatic middle-aged healthy adults with multiple lifestyle and vascular risk measures, as well as neuroimaging.

- Our statistical approach using confirmatory factor analysis and structural equation modelling allowed us to simultaneously discern the independent and inter-related effects of several lifestyle variables and vascular risk factors on brain atrophy and cognition.

- A limitation of the study was its cross-sectional nature. Longitudinal studies with larger samples are necessary to test for temporal and causal inter-relationship between modifiable risk factors and future Alzheimer's disease (AD).

- We caution that participants were carefully screened research participants, which limits our ability to generalise results to the entire population. More studies are needed to replicate these research findings in community-based settings.

- MRI measures are largely seen as biomarkers of neurodegeneration associated with $A D$. However, biomarkers specific to $A D$ pathology (amyloid and tau pathology) are warranted to determine whether the associations between lifestyle and vascular risk are indeed related to ongoing AD.

with larger samples are needed to replicate these research findings in more diverse, community-based settings.

\section{INTRODUCTION}

Alzheimer's disease (AD) is the most common form of dementia, affecting nearly 34 million people worldwide. ${ }^{1}$ Unless effective strategies for prevention are found, the prevalence of $\mathrm{AD}$ is expected to triple by 2050 . 
Table 1 Participants' demographic and clinical characteristics

\begin{tabular}{ll}
$\mathrm{n}$ & 116 \\
\hline Age, years & $50(6)$, range 30-60 \\
\hline Sex, \% female & 62 \\
\hline Education, years & $16(2)$, range 12-22 \\
$\begin{array}{l}\text { Family history of LOAD, \% } \\
\text { positive }\end{array}$ & 38 \\
\hline
\end{tabular}

\begin{tabular}{ll} 
APOE \&4 carriers, \% positive & 40 \\
\hline Ethnicity, \% white & 68 \\
$\begin{array}{l}\text { Subjective complaints, \% } \\
\text { positive }\end{array}$ & 44
\end{tabular}

Laboratory findings

\begin{tabular}{ll} 
BMI (kg/cm $\left.{ }^{2}\right)$ & $25(4)$, range 18-37 \\
Hypertension, \% positive & 14 \\
QUICKI score (unitless) & $0.32(0.03)$ \\
$\begin{array}{l}\text { Plasma cholesterol/HDL ratio } \\
\text { (unitless) }\end{array}$ & $3.3(0.8)$ \\
$\begin{array}{l}\text { Plasma homocysteine ( } \mu \mathrm{mol} / \mathrm{L}) \\
\text { Lifestyle measures }\end{array}$ & \\
$\begin{array}{l}\text { Mediterranean diet (unitless) } \\
\text { Physical activity (metabolic }\end{array}$ & $4.3(1.9)$, range 1-8 \\
$\begin{array}{l}\text { equivalent/hour) } \\
\text { Intellectual activity (unitless) }\end{array}$ & $3.7(0.3)$, range 1-37 \\
\hline
\end{tabular}

Values are mean (SD) unless otherwise specified.

*Data were not available for 20 out of 116 patients.

APOE, apolipoprotein E; BMI, body mass index; HDL, high-density lipoprotein; LOAD, late-onset Alzheimer's disease; QUICKI, Quantitative Insulin Sensitivity Check Index.

Given the current lack of disease-modifying treatment, ${ }^{2}$ and increasing awareness that $\mathrm{AD}$ pathology develops over many years prior to clinical symptoms, ${ }^{3}$ the potential for prevention is crucial to reduce $\mathrm{AD}$ risk and/or delay the onset of cognitive decline.

There is substantial epidemiological evidence linking modifiable risk factors, such as midlife hypertension, obesity, diabetes, poor diet, physical and intellectual inactivity, with increased risk of late-onset $\mathrm{AD}$. Recent population-attributable models estimated that one in every three cases of $\mathrm{AD}$ may be accounted for by these lifestyle and vascular-related risk factors. ${ }^{14}$ As a result, trends in $\mathrm{AD}$ risk reduction research have focused on lifestyle interventions as well as vascular risk reduction. ${ }^{235}$

While the importance of lifestyle and vascular risk management in health is well understood, their specific effects on $\mathrm{AD}$ pathophysiology, and thus their effectiveness for $\mathrm{AD}$ prevention are less clear. ${ }^{6}$

Conflicting results in the literature may be due to the use of different patient populations, different lifestyle measures, different statistical constructs and an overall lack of AD biomarkers. Moreover, though a combined reduction in risk factors is projected to have a greater impact than any one factor alone,${ }^{4}$ most studies have examined one or few variables at a time. ${ }^{7}$ Further, although there is consensus that preventative interventions have the highest chances of success when implemented in midlife, ${ }^{2}$ the majority of studies focused on the elderly, including those already experiencing cognitive impairment. ${ }^{8-14}$

Neuropathologically, AD is characterised by the presence of amyloid-beta $(A \beta)$ plaques, neurofibrillary tangles and neuronal loss in selectively vulnerable brain regions. ${ }^{6}$ Neuronal loss in $\mathrm{AD}$ originates in the medial temporal lobes during the normal stages of cognition and spreads to cortical regions with disease progression. ${ }^{15}$ MRI has long been used to visualise neurodegeneration in vivo. Several MRI studies have shown that brain cortical thinning (ie, atrophy) in AD-vulnerable regions begins many years prior to dementia onset, making this biomarker an ideal candidate to monitor the effectiveness of preventative interventions. ${ }^{16}$

This study examines the simultaneous effects of multiple modifiable lifestyle and vascular risk factors on MRI-based cortical atrophy in a cohort of middle-aged healthy adults at risk for $\mathrm{AD}$.

\section{METHODS \\ Participants}

Participants were enrolled in observational brain imaging studies of clinically and cognitively normal middle-aged adults conducted at two research centres affiliated with the Alzheimer's disease Coordinating Center of New York University (NYU) School of Medicine between 2010 and 2016. Details about the studies have previously been published. ${ }^{17-21}$ All participants provided informed consent to participate in this study.

Briefly, participants were derived from multiple community sources, including individuals interested in research participation, family members and caregivers of impaired patients. Subjects were 30-60years old, with $\geq 12$-year education, Clinical Dementia Rating $=0$, Global Deterioration Scale $<2$, Hamilton depression scale $<16$ and normal cognitive test performance for age and education. ${ }^{22}$ Because our goal was to examine the influence of lifestyle and vascular risk factors in healthy middle-aged participants without overt cardiovascular and/or cerebrovascular disease, we excluded individuals with Modified Hachinski Ischaemia scale score $>4$. Those with medical conditions or history of conditions that may affect brain structure or function (eg, stroke, poorly controlled diabetes, head trauma, any neurodegenerative diseases, depression, hydrocephalus, intracranial mass and infarcts on MRI) and those taking steroids or psychoactive medications were excluded. Only participants with clinical, laboratory, neuropsychological, volumetric MRI examinations and lifestyle questionnaires within 6 months of each other were included.

A family history of AD that included at least one first-degree relative whose $\mathrm{AD}$ onset was after age 60 was elicited using standardised questionnaires. ${ }^{17}$ Apolipoprotein 


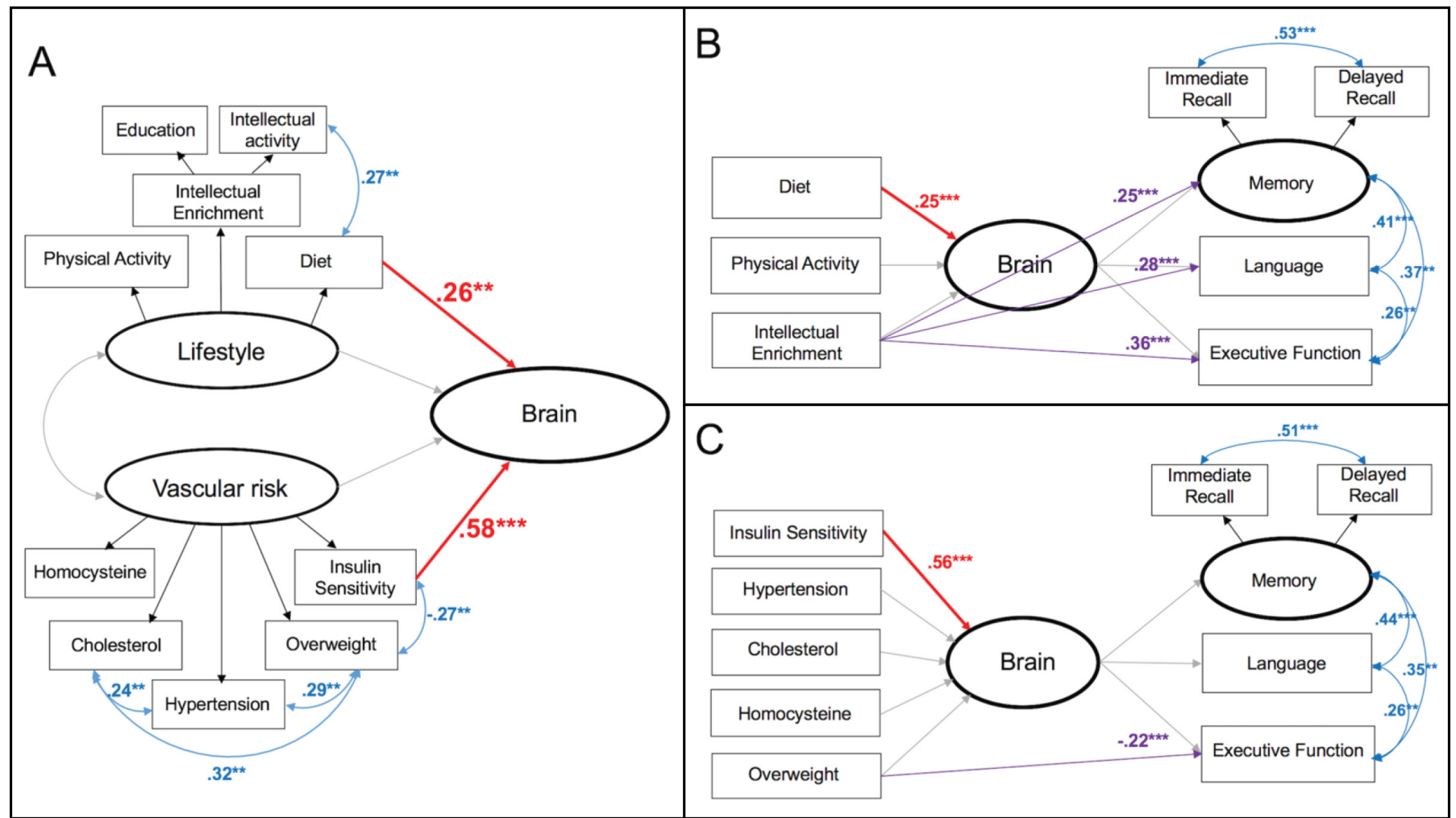

Figure 1 Assessing the impact of lifestyle and vascular risk factors on brain ageing and cognition. Results of the following SEMs. (A) Relationships between lifestyle, vascular risk and brain, excluding cognition from the model. (B) Relationships between lifestyle, brain and cognition, excluding vascular risk from the model. (C) Relationships between vascular risk, brain and cognition, excluding lifestyle from the model. Red lines indicate the significant effects observed in our data. Purple lines indicate significant alternate paths. Blue lines indicate significant correlational effects. Grey lines indicate associations which were tested but found to be non-significant. Numbers beside the lines are standardised beta coefficients and corresponding $P$ values: ${ }^{*} \mathrm{P}<0.01$; ${ }^{*} \mathrm{P}<0.001$. Not shown here, all brain $\mathrm{ROI}$ variables were correlated with one another (see online supplementary etable 1). Direct associations between lifestyle and vascular risk factors are found in online supplementary etable 2. Variables in squares are measured variables (eg, one variable represents this construct). Variables in circles are latent variables (multiple variables were averaged using a CFA to represent that construct). Straight lines indicate causal relationships, curved lines indicate correlational relationships. Age was entered as a covariate. Brain biomarkers were examined with and without adjusting for total intracranial volume. CFA, confirmatory factor analysis; ROI, region of interest; SEMs, structural equation models.

E (APOE) genotypes were determined using standard quantitative PCR procedures. ${ }^{17}$

\section{Cognitive measures}

The neuropsychological battery of tests was previously described. ${ }^{22}$ Briefly, three cognitive domains were assessed from the following tests: memory (immediate and delayed recall of a paragraph and immediate and delayed recall of paired associates), executive function (Wechsler Adult Intelligence Scale (WAIS) digit symbol substitution) and language (WAIS vocabulary).

We modelled memory as a latent factor composed of standardised versions of immediate and delayed recall scores using confirmatory factor analysis (CFA) (alpha $=0.84)$. The three cognitive domains were examined as outcome variables (see Statistical analysis).

\section{Vascular risk measures}

Vascular risk factors included in the model were: (1) overweight, measured using the body mass index (BMI, $\left.\mathrm{kg} / \mathrm{m}^{2}\right)$; (2) presence of hypertension, conservatively determined based on either current antihypertensive treatment or blood pressure assessments; (3) elevated plasma cholesterol and/or elevated plasma homocysteine, as obtained after overnight fasting using standard laboratory procedures and (4) insulin sensitivity, measured with the Quantitative Insulin Sensitivity Check Index, ${ }^{23}$ where lower scores reflect greater insulin resistance and higher scores reflect insulin sensitivity.

\section{Lifestyle measures}

Diet

Dietary data regarding average food consumption over the prior year were obtained using the Block or Harvard food frequency questionnaire, as previously described. ${ }^{19}$ Briefly, food items were categorised into food groups based on similarities in food and nutrient composition, and intake of each food group was calculated by summing the intakes of food group items. Mediterranean diet (MeDi) scores were generated as the sum of caloric intake-adjusted daily gram intake dichotomised 
Table 2 Results of structural equation modelling for model 1: lifestyle, vascular risk and brain

\begin{tabular}{lrrr}
\hline & $\boldsymbol{\beta}_{\mathrm{s}}$ & SE & P value \\
\hline \multicolumn{4}{l}{ Associations between lifestyle and brain MRI biomarkers } \\
Diet $\rightarrow$ Brain & 0.260 & 0.099 & 0.009 \\
Diet $\rightarrow$ Brain & 0.204 & 0.086 & 0.018 \\
Physical activity $\rightarrow$ Brain & -0.130 & 0.108 & \\
Physical activity $\rightarrow$ Brain & -0.058 & 0.047 & \\
Intellectual enrichment $\rightarrow$ & -0.192 & 0.098 & \\
Brain & & & \\
Intellectual enrichment $\rightarrow$ & -0.190 & 0.102 & \\
Brain & & &
\end{tabular}

Associations between vascular risk and brain MRI biomarkers

\begin{tabular}{lrrr} 
Overweight $\rightarrow$ Brain & -0.067 & 0.101 & \\
Overweight $\rightarrow$ Brain & -0.116 & 0.173 & \\
$\begin{array}{l}\text { Insulin sensitivity } \rightarrow \text { Brain } \\
\text { Insulin sensitivity } \rightarrow \text { Brain }\end{array}$ & 0.559 & 0.081 & $<0.001$ \\
$\begin{array}{l}\text { Plasma cholesterol/HDL } \rightarrow \\
\text { Brain }\end{array}$ & -0.038 & 0.101 & \\
$\begin{array}{l}\text { Plasma cholesterol/HDL } \rightarrow \\
\text { Brain }\end{array}$ & -0.010 & 0.037 & \\
$\begin{array}{l}\text { Plasma homocysteine } \rightarrow \\
\text { Brain }\end{array}$ & 0.177 & 0.315 & \\
$\begin{array}{l}\text { Plasma homocysteine } \rightarrow \\
\text { Brain }\end{array}$ & 0.175 & 0.372 & \\
$\begin{array}{l}\text { Hypertension } \rightarrow \text { Brain } \\
\text { Hypertension } \rightarrow \text { Brain }\end{array}$ & 0.076 & 0.106 & \\
\hline
\end{tabular}

The table consists of standardised betas $\left(\beta_{\mathrm{s}}\right)$, their SEs and $\mathrm{P}$ values of the estimates from the full model with age entered as a covariate. The $\beta_{\mathrm{s}}$ can be interpreted as partial correlations. Only significant $P$ values (two tailed) are reported. Paths in italics are adjusted for sex and APOE status as covariates.

APOE, apolipoprotein E; HDL, high-density lipoprotein.

relative to the sex-specific median for each beneficial (fruits, vegetables, legumes, cereals and fish; monounsaturated to saturated fat ratio; mild to moderate alcohol consumption) versus detrimental (meat and dairy products) component. ${ }^{19}$ Greater score indicate greater MeDi adherence.

\section{Physical activity}

The Baecke and Minnesota leisure time physical activity questionnaires were used to estimate the current level of physical activity. ${ }^{25}{ }^{26}$ For each activity, information was collected on the frequency and duration of engagement over the past year. Frequency and duration data were multiplied with an activity-specific intensity code indicating calorie expenditure. We standardised and summed the activity-dependent scores from each test to obtain the overall annual intensity of physical activity per person, which was converted to metabolic equivalents.
Intellectual enrichment

Intellectual activity and years of education were used as measures of intellectual enrichment as described below. Intellectual activity throughout life was assessed using a validated 25-item interview in which participants were asked to report how often they engaged in common cognitively demanding activities that depend minimally on socioeconomic status, such as reading books or newspapers, writing letters or e-mails, going to the library and playing games at different age epochs. ${ }^{9} 27$ Previous studies described this instrument in detail and reported high internal consistency and positive associations of intellectual activity with educational and cognitive performance. ${ }^{27}$

\section{MRI acquisition and processing}

All subjects received three-dimensional volumetric T1-weighted MRI on a 3T scanner according to published protocols. ${ }^{17-21}$ MRIs were acquired and preprocessed as described. ${ }^{19}{ }^{28}$ Volumetric segmentation, cortical surface reconstruction and parcellation were performed using the FreeSurfer V.5.3 software package. ${ }^{29}{ }^{30}$ Cortical thickness measures were obtained for a subset of a priori-defined regions of interest (ROIs) known to show atrophic changes early in $\mathrm{AD}$ and in association with lifestyle ${ }^{7}$ : entorhinal cortex (EC), posterior cingulate cortex (PCC), orbitofrontal cortex, inferior and middle temporal gyrus. FreeSurfer was also used to derive total intracranial volumes (TIV) to adjust for individual variability.

A latent factor of brain structure was generated by combining the ROI thickness measures using CFA to represent that construct. Instead of predicting each of these ROIs individually, the latent factor predicts their shared variance (ie, intercorrelation). EC was set as the marker variable. All ROIs were strongly correlated with each other (alpha $>0.78$, see online supplementary etable 1 ).

\section{Statistical analysis}

We constructed structural equation models (SEMs) (MPlus V. $7^{31}$ ) to evaluate interdependent relationships among the four sets of variables: lifestyle (diet, physical activity and intellectual enrichment), vascular-related risk (overweight, hypertension, insulin resistance, elevated plasma cholesterol and homocysteine levels), MRI biomarkers (latent variable of brain structure) and cognitive measures (latent variable of memory, executive function and language).

The three cognitive domains were modelled as a function of lifestyle variables, vascular variables and MRI biomarkers (see online supplementary efigure 1). Lifestyle and vascular variables were specified as exogenous variables. Brain structure formed an intermediate layer of endogenous variables, and the cognitive domains were the final downstream endogenous variables. As such, exogenous variables (lifestyle and vascular risk measures) predicted brain variables, and brain variables in turn predicted cognition. We regressed brain structure on the lifestyle and vascular variables. We then regressed 
cognitive domains on all other variables in the model. Additionally, ran a separate model in which we replace the latent variable of brain structure with the two limbic regions (ie, EC and PCC) based on evidence of their earlier predictive capacity.

Since variables in the two exogenous domains, lifestyle and vascular risk, were only marginally related to each other (see Results), the model was further broken down into the following three submodels: (1) we first modelled the relationships between lifestyle, vascular risk and brain, excluding cognition from the model; (2) we modelled the relationships between lifestyle, brain and cognition, excluding vascular risk and (3) we modelled the relationships between vascular risk, brain and cognition, excluding lifestyle from the model.

All models were adjusted for the intercorrelations between lifestyle variables, vascular-risk variables, brain structure and cognitive measures as appropriate. Age, gender and APOE status were examined as covariates. Brain measures were evaluated with and without adjustment for TIV.

For each analysis, we first fit the full models, estimating all paths and then assessed the reduced models suggested by the primary analysis (eg, with non-significant paths removed). We used $\chi^{2}$ statistic, comparative fit index (CFI) and root mean square error of approximation (RMSEA) goodness of fit tests to indicate model fits for the full models. Each of these measures incorporates unique criteria to assess fit so a summary of all three measures provides a more comprehensive estimate. After the path coefficients were derived, the paths were thresholded to achieve a second, more parsimonious model, by eliminating paths with $\mathrm{P}$ values $>0.05$. Path elimination was monitored via the $\chi^{2}$, CFI and RMSEA. Good model fit can be reflected by a $\chi^{2}$ to $\mathrm{df}$ ratio $<2.0,{ }^{32}$ a $\mathrm{CFI}>0.85^{33}$ and an RMSEA $<0.06 .{ }^{34}$ The CFI and RMSEA are among the measures least affected by sample size and perform very well at all sample sizes. ${ }^{32}$ As such, CFI and RMSEA were our primary reporting criteria for the reduced models.

Some of the reduced models were saturated (eg, these models accounted for all possible relationships that could exist among variables in our dataset), making overall model fit statistics not applicable. In this case, we focused on specific paths and path significance rather than omnibus measures model fits. The standardised beta coefficients $\left(\beta_{\mathrm{s}}\right)$ can be interpreted as partial correlations. Given that all of the paths are standardised, one can judge meaningfulness by their raw and comparative path weights. All analyses used maximum likelihood estimation in the MPlus package. ${ }^{31}$

\section{RESULTS}

\section{Participants}

A total of 116 participants were included in the analysis (table 1). Participants were on average 50 years old (range $25-60), 62 \%$ women, with education $\geq 12$ years. Of these,
$38 \%$ had a family history of $\mathrm{AD}$, and $40 \%$ had at least one copy of the APOE 4 allele (APOE4 carriers).

\section{Structural equation modelling}

In the full model, age was associated with risk of hypertension $\left(\beta_{\mathrm{s}}=0.18, \mathrm{P}=0.049\right)$ and, although it tended to be negatively related to brain structure $(\beta=-0.10$ to -0.19 , ns), it was not significantly associated with the lifestyle or cognitive variables.

Men, as expected, showed higher vascular risk than women, as reflected in a higher frequency of hypertension, elevated cholesterol and homocysteine levels $(\mathrm{P}<0.04)$. Women showed better executive function performance than men $(\mathrm{P}<0.001)$. Sex was not significantly or marginally related to lifestyle variables or brain structure.

APOE status was not associated with lifestyle or cognitive variables but was associated with plasma cholesterol $(\mathrm{P}=0.033)$ and marginally associated with brain structure $(\mathrm{P}=0.07)$. These effects were driven by APOE4 carriers exhibiting higher cholesterol levels and reduced cortical thickness than non-carriers.

Given these relationships, all subsequent analyses were performed with and without adjusting for age, sex and APOE, as appropriate.

Among lifestyle variables, with and without controlling for the above confounds, participants with higher intellectual enrichment showed higher $\mathrm{MeDi}$ adherence $\left(\beta_{\mathrm{s}} \geq 0.27, \mathrm{P} \leq 0.028\right)$. Otherwise, lifestyle variables were not significantly related to one another.

Among vascular variables, BMI was negatively associated with insulin sensitivity $\left(\beta_{\mathrm{s}}=-0.27, \mathrm{P}<0.01\right)$ and positively associated with cholesterol levels and hypertension $\left(\beta_{\mathrm{s}}=0.32\right.$ and $\left.\beta_{\mathrm{s}}=0.29, \mathrm{P}<0.001\right)$. Cholesterol levels and hypertension were associated with each other $\left(\beta_{\mathrm{s}}=0.24\right.$, $\mathrm{P}<0.05)$.

Vascular and lifestyle variables were not associated with each other, except for higher intellectual enrichment correlating with lower risk of hypertension $\left(\beta_{\mathrm{s}}=-0.22\right.$, $\mathrm{P}<0.05)$, and insulin sensitivity was marginally, though not significantly, associated with $\operatorname{diet}\left(\beta_{\mathrm{s}}=0.12, \mathrm{P}=0.18\right)$. The model was therefore broken down in the following three submodels:

\section{Lifestyle, vascular risk and MRI-based biomarkers}

Figure 1 represents the path diagrams from model 1, which exhibited adequate fit, $\chi^{2}{ }_{(41)} \geq 95.37$, RMSEA $<0.05$, $\mathrm{CFI} \geq 0.85, \mathrm{P}<0.001$. Controlling for age, diet and insulin sensitivity were the only factors positively associated with brain structure $\left(\beta_{\mathrm{s}} \geq 0.26\right.$ and $\beta_{\mathrm{s}} \geq 0.58$, respectively, $\mathrm{P} \leq 0.008$, table 2$)$. The reduced model fit the data well (RMSEA $<0.01$, CFI $\geq 0.90$ ) reflecting the fact that diet and insulin sensitivity both significantly and independently predicted brain structure (diet: $\beta=0.20, \mathrm{P}=0.017$ and insulin sensitivity: $\beta_{\mathrm{s}}=0.57, \mathrm{P}<0.001$ ), consistent with the pattern of significant and non-significant paths obtained in full model 1. Including sex and APOE status as covariates did not significantly influence these relationships (table 2). 


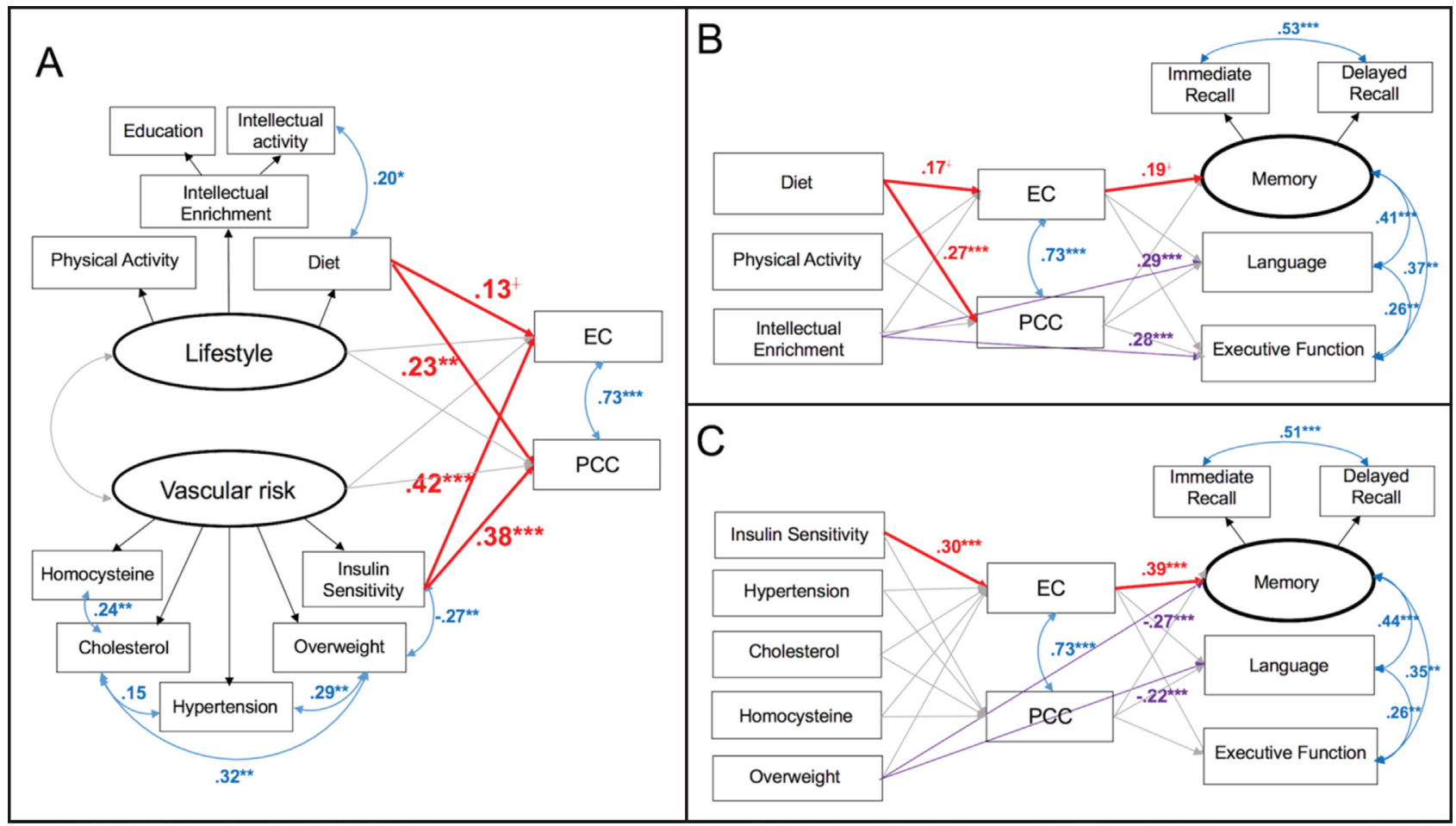

Figure 2 Assessing the impact of lifestyle and vascular risk factors on limbic brain structures and cognition. Results of the following SEMs. (A) Relationships between lifestyle, vascular risk and limbic brain, excluding cognition from the model. (B) Relationships between lifestyle, limbic brain and cognition, excluding vascular risk from the model. (C) Relationships between vascular risk, limbic brain and cognition, excluding lifestyle from the model. See legend to figure 1. Age was entered as a covariate. Brain measures were examined with and without adjusting for total intracranial volume. ${ }^{\star} P<0.01$; ${ }^{\star \star} P<0.001$. EC, entorhinal cortex; PCC, posterior cingulate cortex; SEMs, structural equation models.

Restricting analysis to limbic structures fit the data well (RMSEA $<0.05$, CFI $=1.0$ ), confirming that insulin sensitivity was positively associated with both EC and PCC $\left(\beta_{\mathrm{s}}=0.42\right.$ and $\left.\beta_{\mathrm{s}}=0.38, \mathrm{P}<0.001\right)$, and diet was associated with PCC $(\beta=0.23, P=0.004)$ and marginally with $\mathrm{EC}$ $\left(\beta_{\mathrm{s}}=0.13, \mathrm{P}=0.12\right)$ (figure 2$)$.

\section{Lifestyle, MRI-based biomarkers and cognition}

Model 2 exhibited adequate fit, $\chi^{2}{ }_{(64)} \geq 108.51$, RMSEA $<0.05, \mathrm{CFI} \geq 0.90, \mathrm{P}<0.001$. The reduced models were completely saturated (eg, all possible relationships that could exist among variables in the dataset were accounted for). As such, we focused on specific paths and path significance rather than omnibus measures of model fit.

As in model 1, with and without adjusting for age and TIV, diet was the only lifestyle factor positively associated with brain structure $\left(\beta_{\mathrm{s}} \geq 0.27, \mathrm{P} \leq 0.02\right.$, table 3 and figure 1$)$. Diet and physical activity were not associated with cognitive function, whereas intellectual enrichment was positively associated with cognition $(\mathrm{P}<0.05$, table 3$)$. Specifically, intellectual activity was positively associated with memory and executive function $\left(\beta_{\mathrm{s}}=0.28\right.$ and $\beta_{\mathrm{s}}=0.39$, respectively, $\mathrm{P}<0.02)$, and marginally with language $\left(\beta_{\mathrm{s}}=0.25, \mathrm{P}=0.08\right)$. Education positively predicted memory, executive function and language $\left(\beta_{\mathrm{s}}=0.21-0.33, \mathrm{P} \leq 0.038\right)$.

Brain structure was not significantly associated with cognition. However, when analyses were restricted to limbic structures, diet showed positive associations with
PCC $\left(\beta_{\mathrm{s}}=0.27, \mathrm{P}=0.006\right)$ and EC $\left(\beta_{\mathrm{s}}=0.17, \mathrm{P}=0.08\right)$, which in turn positively, though marginally, predicted memory $\left(\beta_{\mathrm{s}}=0.19, \mathrm{P}=0.078\right)$ (figure 2$)$.

Including sex and APOE status as covariates did not influence the other relationships of interest in any of the models (table 3 ).

\section{Vascular risk, MRI-based biomarkers and cognition}

Model three exhibited adequate fit, $\chi^{2}{ }_{(78)} \geq 151.54$, RMSEA $<0.05, \quad$ CFI $\geq 0.89, \quad \mathrm{P}<0.001$. The reduced models were completely saturated and overall model fit statistics were not applicable. Below, we focus on path significance rather than omnibus measures of model fit.

As in model 1, insulin sensitivity was the only vascular factor associated with brain structure $\left(\beta_{\mathrm{s}}=0.58, \mathrm{P}<0.01\right.$, table 4 and figure 1 ). Brain structure was not significantly associated with cognition. However, when analysis was restricted to limbic structures, insulin sensitivity showed positive associations with EC $\left(\beta_{\mathrm{s}}=0.30, \mathrm{P}<0.001\right)$ and $\mathrm{EC}$ in turn positively predicted memory $(\beta=0.39, \mathrm{P}<0.001)$ (figure 2). Additionally, BMI was negatively associated with memory and executive function $\left(\beta_{\mathrm{s}}=-0.22\right.$ and $\beta_{\mathrm{s}}=-0.27, \mathrm{P} \leq 0.021$, figure 2 ).

Including sex and APOE status as covariates did not significantly influence the other relationships of interest (table 4). 
Table 3 Results of structural equation modelling for model 2: lifestyle, brain and cognition

\begin{tabular}{|c|c|c|c|}
\hline & $\boldsymbol{\beta}_{\mathrm{s}}$ & SE & $P$ value \\
\hline \multicolumn{4}{|c|}{ Associations between lifestyle and brain MRI biomarkers } \\
\hline Diet $\rightarrow$ Brain & 0.253 & 0.098 & 0.010 \\
\hline Diet $\rightarrow$ Brain & 0.253 & 0.099 & 0.010 \\
\hline Physical activity $\rightarrow$ Brain & -0.129 & 0.107 & \\
\hline Physical activity $\rightarrow$ Brain & -0.132 & 0.126 & \\
\hline \multicolumn{4}{|l|}{ Intellectual enrichment $\rightarrow$ Brain } \\
\hline Intellectual activity $\rightarrow$ Brain & -0.113 & 0.125 & \\
\hline Education $\rightarrow$ Brain & -0.164 & 0.099 & \\
\hline \multicolumn{4}{|l|}{ Intellectual enrichment $\rightarrow$ Brain } \\
\hline Intellectual activity $\rightarrow$ Brain & -0.112 & 0.124 & \\
\hline Education $\rightarrow$ Brain & -0.168 & 0.099 & \\
\hline \multicolumn{4}{|c|}{ Associations between brain and cognitive measures } \\
\hline Brain $\rightarrow$ Memory & 0.050 & 0.118 & \\
\hline Brain $\rightarrow$ Memory & 0.064 & 0.094 & \\
\hline Brain $\rightarrow$ Executive function & -0.034 & 0.101 & \\
\hline Brain $\rightarrow$ Executive function & -0.041 & 0.100 & \\
\hline Brain $\rightarrow$ Language & -0.314 & 0.094 & \\
\hline Brain $\rightarrow$ Language & -0.318 & 0.094 & \\
\hline \multicolumn{4}{|c|}{ Associations between lifestyle and cognitive measures } \\
\hline Diet $\rightarrow$ Memory & 0.033 & 0.113 & \\
\hline Diet $\rightarrow$ Memory & 0.023 & 0.092 & \\
\hline Diet $\rightarrow$ Executive function & 0.023 & 0.099 & \\
\hline Diet $\rightarrow$ Executive function & 0.042 & 0.099 & \\
\hline Diet $\rightarrow$ Language & 0.068 & 0.107 & \\
\hline Diet $\rightarrow$ Language & 0.063 & 0.107 & \\
\hline Physical activity $\rightarrow$ Memory & 0.070 & 0.113 & \\
\hline Physical activity $\rightarrow$ Memory & 0.052 & 0.094 & \\
\hline Physical activity $\rightarrow$ Executive function & 0.002 & 0.099 & \\
\hline Physical activity $\rightarrow$ Executive function & 0.007 & 0.098 & \\
\hline Physical activity $\rightarrow$ Language & 0.002 & 0.102 & \\
\hline Physical activity $\rightarrow$ Language & -0.009 & 0.102 & \\
\hline Intellectual enrichment $\rightarrow$ Memory & 0.278 & 0.114 & 0.014 \\
\hline \multicolumn{4}{|l|}{ Intellectual activity $\rightarrow$ Memory } \\
\hline Education $\rightarrow$ Memory & 0.213 & 0.102 & 0.038 \\
\hline \multicolumn{4}{|l|}{$\begin{array}{l}\text { Intellectual enrichment } \rightarrow \text { Executive } \\
\text { function }\end{array}$} \\
\hline Intellectual activity $\rightarrow$ Executive function & 0.385 & 0.101 & $<0.001$ \\
\hline Education $\rightarrow$ Executive function & 0.329 & 0.084 & $<0.001$ \\
\hline \multicolumn{4}{|l|}{ Intellectual enrichment $\rightarrow$ Language } \\
\hline Intellectual activity $\rightarrow$ Language & 0.251 & 0.135 & \\
\hline Education $\rightarrow$ Language & 0.325 & 0.084 & $<0.001$ \\
\hline Intellectual enrichment $\rightarrow$ Memory & 0.184 & 0.094 & 0.050 \\
\hline $\begin{array}{l}\text { Intellectual enrichment } \rightarrow \text { Executive } \\
\text { function }\end{array}$ & 0.327 & 0.084 & 0.001 \\
\hline Intellectual enrichment $\rightarrow$ Language & 0.326 & 0.084 & 0.001 \\
\hline
\end{tabular}

The table consists of standardised betas ( $\beta \mathrm{s}$ ), their SEs, and $\mathrm{P}$ values of the estimates from the full model with age entered as a covariate. The $\beta$ s can be interpreted as partial correlations. Only significant $P$ values (two tailed) are reported. Paths in italics are adjusted for sex and APOE status as covariates.
Table 4 Results of structural equation modelling for model 3: vascular risk, brain and cognition

\begin{tabular}{lrll}
\hline & \multicolumn{1}{c}{$\boldsymbol{\beta}_{\mathrm{s}}$} & SE & P value \\
\hline $\begin{array}{l}\text { Associations between vascular risk and brain MRI } \\
\text { biomarkers }\end{array}$ & & \\
\hline Overweight $\rightarrow$ Brain & -0.067 & 0.101 & \\
Overweight $\rightarrow$ Brain & -0.116 & 0.173 & \\
Insulin sensitivity $\rightarrow$ Brain & 0.559 & 0.081 & $<0.001$ \\
Insulin sensitivity $\rightarrow$ Brain & 0.029 & 0.006 & $<0.001$ \\
Plasma cholesterol/HDL $\rightarrow$ Brain & -0.038 & 0.101 & \\
Plasma cholesterol/HDL $\rightarrow$ Brain & -0.010 & 0.037 & \\
Plasma homocysteine $\rightarrow$ Brain & 0.177 & 0.315 & \\
Plasma homocysteine $\rightarrow$ Brain & 0.175 & 0.372 & \\
Hypertension $\rightarrow$ Brain & 0.076 & 0.106 & \\
Hypertension $\rightarrow$ Brain & 0.032 & 0.042 & \\
\hline Associations & & &
\end{tabular}

Associations between brain and cognitive measures

$\begin{array}{lrr}\text { Brain } \rightarrow \text { Memory } & 0.032 & 0.125 \\ \text { Brain } \rightarrow \text { Memory } & 0.042 & 0.123 \\ \text { Brain } \rightarrow \text { Executive function } & -0.032 & 0.101 \\ \text { Brain } \rightarrow \text { Executive function } & -0.037 & 0.101 \\ \text { Brain } \rightarrow \text { Language } & -0.325 & 0.095 \\ \text { Brain } \rightarrow \text { Language } & -0.327 & 0.095\end{array}$

Associations between vascular risk and cognitive measures

Overweight $\rightarrow$ Memory $\quad-0.195 \quad 0.119$

Overweight $\rightarrow$ Memory $\quad-0.191 \quad 0.119$

Overweight $\rightarrow$ Executive function $\quad-0.230 \quad 0.091$

0.012

Overweight $\rightarrow$ Executive function $\quad-0.229 \quad 0.091$

0.012

Overweight $\rightarrow$ Language $\quad-0.006 \quad 0.095$

Overweight $\rightarrow$ Language $\quad-0.008 \quad 0.095$

Insulin sensitivity $\rightarrow$ Memory $\quad 0.082 \quad 0.116$

Insulin sensitivity $\rightarrow$ Memory $\quad 0.041 \quad 0.124$

Insulin sensitivity $\rightarrow$ Executive $\quad-0.072 \quad 0.097$

function

\begin{tabular}{|c|c|c|c|}
\hline $\begin{array}{l}\text { Insulin sensitivity } \rightarrow \text { Executive } \\
\text { function }\end{array}$ & -0.064 & 0.096 & \\
\hline Insulin sensitivity $\rightarrow$ Language & -0.480 & 0.075 & $<0.001$ \\
\hline Insulin sensitivity $\rightarrow$ Language & -0.490 & 0.074 & $<0.001$ \\
\hline Plasma homocysteine $\rightarrow$ Memory & -0.298 & 0.164 & \\
\hline Plasma homocysteine $\rightarrow$ Memory & -0.183 & 0.196 & \\
\hline $\begin{array}{l}\text { Plasma homocysteine } \rightarrow \text { Executive } \\
\text { function }\end{array}$ & -0.190 & 0.140 & \\
\hline $\begin{array}{l}\text { Plasma homocysteine } \rightarrow \text { Executive } \\
\text { function }\end{array}$ & -0.188 & 0.122 & \\
\hline Plasma homocysteine $\rightarrow$ Language & -0.218 & 0.338 & \\
\hline Plasma homocysteine $\rightarrow$ Language & -0.358 & 0.294 & \\
\hline Cholesterol/HDL $\rightarrow$ Memory & -0.051 & 0.115 & \\
\hline Cholesterol/HDL $\rightarrow$ Memory & -0.042 & 0.112 & \\
\hline $\begin{array}{l}\text { Cholesterol/HDL } \rightarrow \text { Executive } \\
\text { function }\end{array}$ & -0.138 & 0.097 & \\
\hline $\begin{array}{l}\text { Cholesterol/HDL } \rightarrow \text { Executive } \\
\text { function }\end{array}$ & -0.030 & 0.101 & \\
\hline Cholesterol/HDL $\rightarrow$ Language & -0.057 & 0.096 & \\
\hline
\end{tabular}

Continued 


\begin{tabular}{llll} 
Table 4 Continued & & & \\
\hline & $\boldsymbol{\beta}_{\mathrm{s}}$ & SE & P value \\
\hline Cholesterol/HDL $\rightarrow$ Language & -0.052 & 0.095 & \\
Hypertension $\rightarrow$ Memory & -0.084 & 0.129 & \\
Hypertension $\rightarrow$ Memory & -0.084 & 0.130 & \\
Hypertension $\rightarrow$ Executive function & -0.092 & 0.099 & \\
Hypertension $\rightarrow$ Executive function & -0.073 & 0.098 & \\
Hypertension $\rightarrow$ Language & -0.176 & 0.095 & \\
Hypertension $\rightarrow$ Language & -0.179 & 0.094 & \\
\hline
\end{tabular}

The table consists of standardised betas ( $\beta \mathrm{s})$, their $\mathrm{SEs}$, and $\mathrm{P}$ values of the estimates from the full model with age entered as a covariate. The $\beta$ s can be interpreted as partial correlations. Only significant $P$ values (two tailed) are reported. Paths in italics are adjusted for sex and APOE status as covariates.

$\mathrm{HDL}$, high-density lipoprotein.

\section{Discussion}

The major conclusions of this study were the following: (1) among lifestyle and vascular risk factors, diet and insulin sensitivity explained variability in brain cortical thickness in cognitively healthy, middle-aged adults; (2) MRI measures of limbic structures in turn explained variability in memory performance; (3) intellectual enrichment and increased BMI explained variability in cognitive performance through different pathways.

Our results indicate that diet and insulin sensitivity may be among the earliest modifiable risk factors to influence the expression of AD biomarkers, suggesting these modifiable risk factors may alter risk of $\mathrm{AD}$ pathophysiology during middle age. These associations were independent of age, sex and APOE genotype.

Findings of associations between lower MeDi adherence and increased brain atrophy are consistent with the literature in the elderly. ${ }^{19243536}$ Likewise, we and others have described strong associations between insulin resistance and limbic cortex atrophy in both adolescents and non-demented elderly. ${ }^{20237}$ Our data extend prior observations to a population of middle-aged healthy adults and offers a comprehensive view of how the interplay of lifestyle and vascular factors influences possible $\mathrm{AD}$ risk. Indeed, besides being linked with lower risk of dementia, the MeDi was shown to support healthier insulin regulation and cardiovascular health. ${ }^{38}$ Given the known increased $\mathrm{AD}$ risk associated with prediabetes and type 2 diabetes, ${ }^{39}$ our findings fit with increased cardiovascular risk being a driver of increased brain ageing and AD.

The statistical approach we employed allowed us to simultaneously examine several lifestyle and vascular risk factors and discern their independent as well as intercorrelated effects on brain atrophy and cognition. Other studies that used SEM reported minimal or null associations between physical and intellectual activities and $\mathrm{AD}$ biomarkers in non-demented elderly, although biomarkers independently predicted cognitive function. ${ }^{1240}$ These studies, however, did not take into account diet or vascular risk. Our study in a younger cohort (mean age 50 vs $78-82$ years ${ }^{12} 40$ ) indicates that physical and intellectual activity do not impact brain ageing as much as diet does during the normal stages of cognition. When vascular risk factors were accounted for in the model, insulin sensitivity influences cortical thickness and its effects on limbic regions had significant effects on memory performance. These effects were present accounting for the impact of exercise, intellectual activity and additional vascular risk factors.

In our cohort, insulin resistance was strongly associated with increased BMI (ie, overweight). Although BMI was not directly associated with MRI measures, it negatively impacted memory and executive function through other pathways. It has long been known that midlife obesity affects cognitive performance, ${ }^{41}$ possibly by promoting cardiovascular disease (CVD) and A $\beta$ deposition. ${ }^{42}$

Additionally, intellectual enrichment was associated with better cognitive performance, suggesting a protective effect on $\mathrm{AD}$ risk. It is possible that continued intellectual stimulation may lower the risk or delay the onset of $\mathrm{AD}$ by enhancing cognitive reserve, as suggested by studies in the elderly. $911-1440$

Several issues require further consideration. First, our results are cross-sectional and do not allow for determination of causality or temporal relationships between lifestyle, vascular risk, brain biomarkers and cognitive status. Studies with larger samples and longitudinal follow-ups are needed to examine lifestyle and vascular risk factors as possible $\mathrm{AD}$ risk modifiers. From a statistical perspective, even though our model fits were adequate and the observed relationships were supported by the data, the associations were not so strong to yield an ideal model fit. To better evaluate the impact of modifiable risk factors on brain ageing, we performed additional linear regression analyses to estimate R-squared values for the predictors identified in the reduced SEM models. R-squared is the 'per cent of variance explained' by the model (eg, the fraction by which the variance of the errors is less than the variance of the dependent variable). As with SEM analysis, diet and insulin sensitivity were both significant predictors of brain structure, yielding a combined R-squared $=0.28$ (age adjusted), $\mathrm{P}<0.001$. In other words, the combination of diet and insulin sensitivity explained $28 \%$ of the variance in brain MRI measures. Additionally, intellectual enrichment and BMI explained $11 \%$ and $8 \%$ of the variance in cognitive measures, respectively $(\mathrm{P} \leq 0.022)$. As such, these modifiable risk factors were fairly good predictors after adjusting for all other variables in the model. The associations were nonetheless modest, which is not unexpected in a relatively young, healthy population. That said, caution should be exerted in interpreting these data. For instance, some of the null associations may depend on sample size limitations. However, null effects observed in our cohort are consistent with negative findings from large-scale studies in the elderly. ${ }^{12} 40$ Therefore, we offer that the strongest arguments of the study are the significant findings which manifest themselves despite these limitations. Overall, 
our data suggest that lifestyle and vascular risk have an impact on brain ageing during midlife, thus supporting further investigation of modifiable risk factors for healthy brain ageing and $\mathrm{AD}$ risk reduction.

As with other studies in asymptomatic at-risk individuals, ${ }^{184344}$ imaging biomarkers were only modestly associated with cognitive measures, most likely because our patients were all cognitively normal and younger than 60. Previous studies have demonstrated that associations between brain biomarkers and cognition are evident in clinical populations, such as those with clear brain pathology but not among normal populations. ${ }^{45}$ Nonetheless, associations between limbic MRI measures and memory were significant, indicating that modifiable risk factors may impact cognitive health before old age.

We cannot exclude that our screening criteria may have biased effect estimates. ${ }^{46}$ For instance, participation was limited to healthy, middle-aged individuals without severe cardiac and CVD. Our goal was to identify possible vascular, metabolic and lifestyle correlates of brain health, prior to severe disease and at a young enough age for potential interventions to be impactful. However, this makes our population restricted in the variability of cardiometabolic disease. As such, our results are only relevant to middle-aged, cognitively normal, healthy men and women without active CVD, stroke or diabetes. Nonetheless, our results are in keeping with prospective, community-based studies of non-diabetic populations showing that insulin resistance and increased blood sugar levels, even at levels considered normal in standard glucose tests, increase $\mathrm{AD}$ risk. ${ }^{47}$

Another limitation of the study pertains to the characterisation of lifestyle habits. We used self-report questionnaires of diet and lifestyle, which are vulnerable to error and may not have captured relevant dimensions of lifestyle activities that influence cognitive functioning. Additionally, given the cross-sectional nature of our study and synchronous timing of lifestyle and MRI assessments, we cannot exclude the possibility that dietary adherence or physical activity levels were short-term choices in this cohort, which are less likely to impact brain biomarkers than long-term choices. However, 90\% of participants reported living the lifestyle assessed in the surveys for 5 years or more.

Although MRI measures of neurodegeneration are sensitive to early $\mathrm{AD}$ changes, they are believed to emerge after changes in neuronal activity and downstream to $A \beta$ accumulation. ${ }^{16}$ As such, MRI measures are not specific to $\mathrm{AD}$ and offer limited information on whether the observed associations were due to $\mathrm{AD}$ or to other causes of cortical thinning. Amyloid and tau biomarkers specific to $\mathrm{AD}$ are warranted to investigate the potential of lifestyle and vascular risk intervention for $A D$ prevention. However, $A \beta$ deposition is an age-dependent phenomenon, with $0 \%$ of cognitively normal individuals aged 45-49 years and less than 6\% of those in the fifth decade of life testing positive for
$\mathrm{A} \beta .^{48}$ Considering that all our participants were cognitively normal and between 30 and 60 years of age, very few (if any) would have had substantial amyloid burden, making this cohort an ideal population for testing of primary prevention strategies.

We caution that present results were found in small numbers of carefully screened patients who were evaluated under controlled clinical conditions. Longitudinal studies with larger samples are needed to replicate and assess the generalisability of these preliminary findings in community-based populations with higher variability in socioeconomic and medical status, as well as to incorporate other AD biomarkers. Should preventative studies prove successful, work will be needed to estimate the effects of increased longevity on dementia burden in such an increasingly older, although healthier, population.

In conclusion, our results suggest that a well-rounded lifestyle that incorporates a healthy diet (such as the $\mathrm{MeDi}$ ), reduces vascular risk factors (especially insulin resistance and overweight) and promotes intellectual activity might be neuroprotective during ageing. More studies are needed to evaluate midlife lifestyle and vascular risk factor modification for $\mathrm{AD}$ prevention.

\section{Author affiliations}

${ }^{1}$ Department of Neurology, Weill Cornell Medical College, New York City, New York, USA

${ }^{2}$ Department of Psychiatry, New York University School of Medicine, New York City, New York, USA

${ }^{3}$ Department of Nutrition and Food Studies, New York University Steinhardt School of Public Health, New York City, New York, USA

${ }^{4}$ Department of Psychology, New York University, New York City, New York, USA

${ }^{5}$ ADM Diagnostics, Chicago, Illinois, USA

${ }^{6}$ Hunter-Bellevue School of Nursing, Hunter College, The City University of New York, New York City, New York, USA

Contributors LM, JS and Rl: study concept and design. LM, CQ, PM, MJDL, AC and RS0: acquisition of data. LM, MW, JS, CQ, REA, DCM, CG and AC: analysis and interpretation. All authors: critical revision of the manuscript for important intellectual content. AC: study supervision.

Funding This study was supported by NIH/NIA grants AG035137, AG13616, 2P01AG026572 and NIH/NIDDK DK083537; funding from the Department of Neurology at Weill Cornell Medical College and philanthropic support of the Alzheimer's Prevention Clinic, Weill Cornell Memory Disorders Program.

Competing interests None declared.

Patient consent Obtained.

Ethics approval This study was approved by NYU School of Medicine Institutional Review Board.

Provenance and peer review Not commissioned; externally peer reviewed.

Data sharing statement All relevant data have been included in the paper. Technical appendix, statistical code and dataset will be made available on request.

Open Access This is an Open Access article distributed in accordance with the Creative Commons Attribution Non Commercial (CC BY-NC 4.0) license, which permits others to distribute, remix, adapt, build upon this work non-commercially, and license their derivative works on different terms, provided the original work is properly cited and the use is non-commercial. See: http://creativecommons.org/ licenses/by-nc/4.0/

(c) Article author(s) (or their employer(s) unless otherwise stated in the text of the article) 2018. All rights reserved. No commercial use is permitted unless otherwise expressly granted. 


\section{REFERENCES}

1. Barnes DE, Yaffe K. The projected effect of risk factor reduction on Alzheimer's disease prevalence. Lancet Neurol 2011;10:819-28.

2. Andrieu S, Coley N, Lovestone S, et al. Prevention of sporadic Alzheimer's disease: lessons learned from clinical trials and future directions. Lancet Neurol 2015;14:926-44.

3. Sperling RA, Karlawish J, Johnson KA. Preclinical Alzheimer disease: the challenges ahead. Nat Rev Neurol 2013;9:54-8.

4. Norton S, Matthews FE, Barnes DE, et al. Potential for primary prevention of Alzheimer's disease: an analysis of population-based data. Lancet Neurol 2014;13:788-94.

5. Biessels GJ. Capitalising on modifiable risk factors for Alzheimer's disease. Lancet Neurol 2014;13:752-3.

6. Mattson MP. Late-onset dementia: a mosaic of prototypical pathologies modifiable by diet and lifestyle. NPJ Aging Mech Dis $2015 ; 1$.

7. Mosconi L, McHugh PF. Let food be thy medicine: diet, nutrition, and biomarkers' risk of Alzheimer's disease. Curr Nutr Rep 2015;4:126-35.

8. Head D, Bugg JM, Goate AM, et al. Exercise engagement as a moderator of the effects of APOE genotype on amyloid deposition. Arch Neurol 2012;69:636-43.

9. Landau SM, Marks SM, Mormino EC, et al. Association of lifetime cognitive engagement and low $\beta$-amyloid deposition. Arch Neurol 2012;69:623-9.

10. Liang KY, Mintun MA, Fagan AM, et al. Exercise and Alzheimer's disease biomarkers in cognitively normal older adults. Ann Neurol 2010;68:311-8.

11. Merrill DA, Siddarth $P$, Raji CA, et al. Modifiable risk factors and brain positron emission tomography measures of amyloid and tau in nondemented adults with memory complaints. Am J Geriatr Psychiatry 2016;24:729-37.

12. Vemuri P, Lesnick TG, Przybelski SA, et al. Effect of intellectual enrichment on $A D$ biomarker trajectories: longitudinal imaging study. Neurology 2016;86:1128-35.

13. Vemuri P, Lesnick TG, Przybelski SA, et al. Association of lifetime intellectual enrichment with cognitive decline in the older population. JAMA Neurol 2014;71:1017-24.

14. Wirth M, Haase CM, Villeneuve S, et al. Neuroprotective pathways: lifestyle activity, brain pathology, and cognition in cognitively normal older adults. Neurobiol Aging 2014;35:1873-82.

15. Morrison JH, Hof PR. Life and death of neurons in the aging brain. Science 1997;278:412-9.

16. Jack CR, Knopman DS, Jagust WJ, et al. Tracking pathophysiological processes in Alzheimer's disease: an updated hypothetical model of dynamic biomarkers. Lancet Neurol 2013;12:207-16.

17. Mosconi L, Brys M, Switalski R, et al. Maternal family history of Alzheimer's disease predisposes to reduced brain glucose metabolism. Proc Natl Acad Sci U S A 2007;104:19067-72.

18. Mosconi L, Murray J, Davies M, et al. Nutrient intake and brain biomarkers of Alzheimer's disease in at-risk cognitively normal individuals: a cross-sectional neuroimaging pilot study. BMJ Open 2014;4:e004850.

19. Mosconi L, Murray J, Tsui WH, et al. Mediterranean diet and magnetic resonance imaging-assessed brain atrophy in cognitively normal individuals at risk for Alzheimer's disease. J Prev Alzheimers Dis 2014;1:23-32.

20. Convit A, Wolf OT, Tarshish C, et al. Reduced glucose tolerance is associated with poor memory performance and hippocampal atrophy among normal elderly. Proc Natl Acad Sci U S A 2003;100:2019-22.

21. Frosch $\mathrm{OH}$, Yau PL, Osorio RS, et al. Insulin resistance among obese middle-aged is associated with decreased cerebrovascular reactivity. Neurology 2017;89:249-55.

22. De Santi S, Pirraglia E, Barr W, et al. Robust and conventional neuropsychological norms: diagnosis and prediction of age-related cognitive decline. Neuropsychology 2008;22:469-84.

23. Katz A, Nambi SS, Mather K, et al. Quantitative insulin sensitivity check index: a simple, accurate method for assessing insulin sensitivity in humans. J Clin Endocrinol Metab 2000;85:2402-10.
24. Gu Y, Brickman AM, Stern $Y$, et al. Mediterranean diet and brain structure in a multiethnic elderly cohort. Neurology 2015;85:1744-51.

25. Taylor HL, Jacobs DR, Schucker B, et al. A questionnaire for the assessment of leisure time physical activities. J Chronic Dis 1978:31:741-55.

26. Baecke JA, Burema J, Frijters JE. A short questionnaire for the measurement of habitual physical activity in epidemiological studies. Am J Clin Nutr 1982;36:936-42.

27. Wilson R, Barnes L, Bennett D. Assessment of lifetime participation in cognitively stimulating activities. J Clin Exp Neuropsychol 2003;25:634-42.

28. Yau PL, Castro MG, Tagani A, et al. Obesity and metabolic syndrome and functional and structural brain impairments in adolescence. Pediatrics 2012;130:e856-e864.

29. Fischl B, Dale AM. Measuring the thickness of the human cerebral cortex from magnetic resonance images. Proc Natl Acad Sci U S A 2000;97:11050-5.

30. Holland D, Brewer JB, Hagler DJ, et al. Subregional neuroanatomical change as a biomarker for Alzheimer's disease. Proc Natl Acad Sci U S A 2009;106:20954-9.

31. Muthén LK, Muthén BO. Mplus user's guide. 6th edn. Los Angeles, CA: Muthén \& Muthén, 2007.

32. Ullman JB. Structural equation modeling. Tabachnik LSF BG, ed. Using multivariate statistics. needham heights. Boston: Allyn \& Bacon, 2001.

33. Bentler PM. Comparative fit indexes in structural models. Psychol Bull 1990;107:238-46.

34. Hu Li-tze, Bentler PM. Cutoff criteria for fit indexes in covariance structure analysis: Conventional criteria versus new alternatives. Structural Equation Modeling: A Multidisciplinary Journal 1999;6:1-55

35. Luciano M, Corley J, Cox SR, et al. Mediterranean-type diet and brain structural change from 73 to 76 years in a Scottish cohort. Neurology 2017:88:449-55.

36. Scarmeas N, Stern Y, Mayeux R, et al. Mediterranean diet and mild cognitive impairment. Arch Neurol 2009;66.

37. Gold SM, Dziobek I, Sweat V, et al. Hippocampal damage and memory impairments as possible early brain complications of type 2 diabetes. Diabetologia 2007;50:711-9.

38. Esposito K, Maiorino MI, Bellastella G, et al. A journey into a mediterranean diet and type 2 diabetes: a systematic review with meta-analyses. BMJ Open 2015:5:e008222.

39. Whitmer RA, Sidney S, Selby J, et al. Midlife cardiovascular risk factors and risk of dementia in late life. Neurology 2005;64:277-81.

40. Vemuri P, Lesnick TG, Przybelski SA, et al. Effect of lifestyle activities on Alzheimer disease biomarkers and cognition. Ann Neurol 2012;72:730-8

41. Baumgart M, Snyder HM, Carrillo MC, et al. Summary of the evidence on modifiable risk factors for cognitive decline and dementia: a population-based perspective. Alzheimers Dement 2015:11:718-26.

42. Gottesman RF, Schneider AL, Zhou Y, et al. Association between midlife vascular risk factors and estimated brain amyloid deposition. JAMA 2017;317:1443-50

43. Reiman EM, Chen K, Liu X, et al. Fibrillar amyloid-beta burden in cognitively normal people at 3 levels of genetic risk for Alzheimer's disease. Proc Natl Acad Sci U S A 2009;106:6820-5.

44. Vlassenko AG, Mintun MA, Xiong C, et al. Amyloid-beta plaque growth in cognitively normal adults: longitudinal [11C]Pittsburgh compound B data. Ann Neurol 2011;70:857-61.

45. Van Petten C. Relationship between hippocampal volume and memory ability in healthy individuals across the lifespan: review and meta-analysis. Neuropsychologia 2004;42:1394-413.

46. Weuve J, Proust-Lima C, Power MC, et al. Guidelines for reporting methodological challenges and evaluating potential bias in dementia research. Alzheimers Dement 2015;11:1098-109.

47. Crane PK, Walker R, Hubbard RA, et al. Glucose levels and risk of dementia. N Engl J Med 2013;369:540-8.

48. Morris JC, Roe CM, Xiong C, et al. APOE predicts amyloid-beta but not tau Alzheimer pathology in cognitively normal aging. Ann Neurol 2010;67:122-31. 